\title{
PID Controllers Tuning with MCSFilter
}

\author{
Andrey Romanenko ${ }^{1,2}$, Florbela P. Fernandes ${ }^{3, a)}$ and Natércia C.P. Fernandes ${ }^{2}$
}

\author{
a)Corresponding author: fflor@ipb.pt \\ ${ }^{1}$ Ciengis, SA, Coimbra, Portugal \\ ${ }^{2}$ CIEPQPF, Department of Chemical Engineering, University of Coimbra, Portugal \\ ${ }^{3}$ Research Centre in Digitalization and Intelligent Robotics (CeDRI), Instituto Politécnico de Bragança, Portugal
}

\begin{abstract}
This work concerns the tuning of industrial controllers through the use of the Multistart Coordinate Search Filter algorithm. The task is laid as a bounded nonlinear global optimization problem aiming at achieving the best possible performance of the control loops at the lowest possible wearing of the costly valves. Moreover, the tuning was successfully carried out both with and without taking into consideration the existing interactions between different loops.
\end{abstract}

\section{INTRODUCTION}

Proportional-Integral-Derivative (PID) controllers are the most widely used controllers in industry $[1,2,3]$ and expectedly will continue to be the main workhorse of process control [4]. Either performing individually or as a part of more complex control strategies (such as cascade structures, for example), PID controllers are pivotal in the industry. Their function consists of issuing an order to the final control elements (usually valves) so that the process is stimulated in a way that pushes the variables under control to the desired values (setpoints). Such controller order relies on the comparison between the measured value of the controlled variable and its corresponding setpoint. Figure 1 illustrates the situation of a system subjected to the action of a feedback controller.

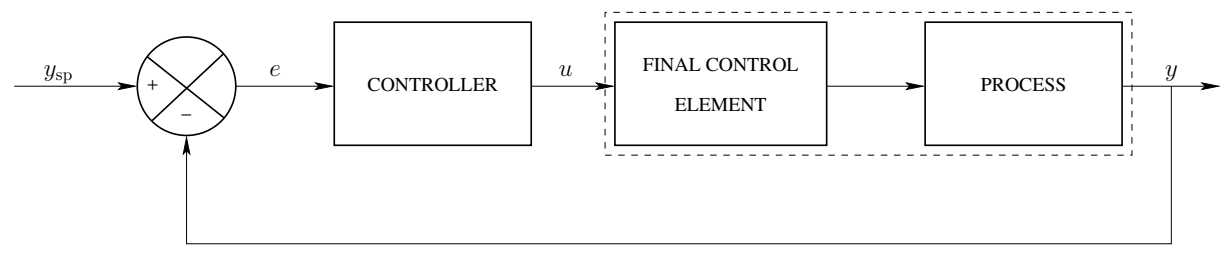

FIGURE 1. Block diagram of a closed control loop.

However, PID controllers need to be tuned in order to actually achieve an effective control of the process rather than contributing to the degradation of the closed loop performance.

The methods used for PID tuning have suffered a remarkable evolution since the unsystematic trial and error. Ziegler and Nichols [5] and Cohen and Coon [6] introduced the first sets of empirical rules that endured over time and are still applied today. Meanwhile, different improvements of these rules and more sophisticated methods have been suggested (for example, Luyben [7], Hang, Åström, and Ho [8], Schei [9], Palmor, Halevi, and Krasney [10]). The most recent class of methods is based in optimization techniques as the work developed by Dittmar et al. [11]. Seeking better methods for tuning PID controllers continues to be an objective to pursue in the process control field, as a more efficient method to tune such widespread controllers may have a worldwide significant impact.

The tuning problem gets especially challenging in the case of mutual dependencies among control loops, as the action needed for controlling a certain variable can have a negative effect in the control of another. A system in which each of its input variables affects only one output variable is called a Single-Input-Single-Output (SISO) system. In 
opposition, if each input variable affects more than one output variable, the system is designated as Multiple-InputMultiple-Output (MIMO). Figure 2 shows schematically these concepts for $2 \times 2$ systems (two input variables and two output variables).
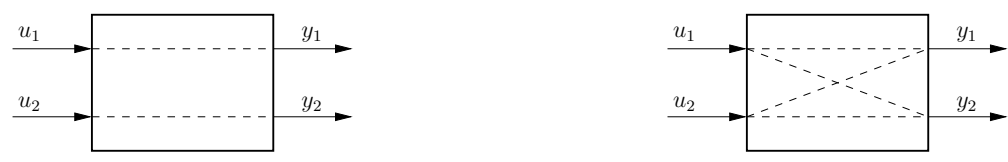

FIGURE 2. Schematic representations of a SISO and a MIMO systems.

The work reported here consists of casting the tuning task as an optimization problem to be solved by the MCSFilter. The MCSFilter is a global method that is able to identify multiple minima. It is a derivative-free method based on a multistart strategy coupled with a local coordinate search filter procedure to find all the minima of a multimodal problem, particularly, the global minimum. The multistart strategy is related to the exploration feature of the method and a derivative-free local search is related to the exploitation of promising regions. Further details about the algorithm are given in [12]. The multistart strategy is based on regions of attraction of all the obtained minimizers.

\section{PROBLEM FORMULATION}

The process to be controlled can be represented by the set of ordinary differential equations (ODE)

$$
\dot{y}(t)=A y(t)+B u(t)
$$

where $y$ represents the output variables vector and $u$ the input variables vector which comprises the stimuli to the system; A and B are matrices that characterize the system.

At each time instant $k$, each controller is in charge of keeping an output variable $i$ as close as possible to its setpoint $\left(y_{i, k}\right.$ and $y_{\mathrm{sp}, i, k}$, respectively), by issuing an order to a final element of control based on the comparison between the actual value of the variable and the desired one. For each control loop $i$ and each time instant $k$, that, for example according to Seborg, Edgar, and Mellichamp [13], is achieved by

$$
u_{i, k}=u_{i, k-1}+K_{\mathrm{c}, i}\left[\left(e_{i, k}-e_{i, k-1}\right)+\frac{\Delta t}{\tau_{\mathrm{I}, i}} e_{i, k}+\frac{\tau_{\mathrm{D}, i}}{\Delta t}\left(e_{i, k}-2 e_{i, k-1}+e_{i, k-2}\right)\right],
$$

where the error is given by $e_{i, k}=y_{\mathrm{sp}, i, k}-y_{i, k} ; K_{\mathrm{c}, i}, \tau_{\mathrm{D}, i}$, and $\tau_{\mathrm{I}, i}$ are the controller's parameters for loop $i$, and $\Delta t$ is the constant sampling time increment.

In order to perform the calculations (2) which will generate adequate stimulation of the system, the controllers parameters have to be known. The estimation of these parameters is the so-called "controller tuning". The tuning problem is cast at the present work as a bounded nonlinear optimization problem, expressed mathematically by

$$
\begin{aligned}
& \min _{P} J(P)=\sum_{k=1}^{n} \sum_{i=1}^{m} w_{1, i}\left(y_{\mathrm{sp}, i, k}-y_{i, k}(P)\right)^{2}+w_{2, i}\left(u_{i, k}(P)-u_{i, k-1}(P)\right)^{2}, \\
& \text { s.t. } \quad P_{\mathrm{L}} \leq P \leq P_{\mathrm{U}}
\end{aligned}
$$

where $P$ is the vector containing the three parameters per controller, $P_{\mathrm{L}}$ and $P_{\mathrm{U}}$ are the lower and upper bounds, and $w_{1}$ and $w_{2}$ are the weights attributed to the mismatch between the actual values and the desired ones for the $m$ variables to be controlled and the intensity of the change along time imposed to the final control elements, respectively. It is noteworthy that such design of the objective function balances the tightness of the setpoint tracking with the degree of aggressiveness of the controller, since the excessive motion of the final control elements provokes its fast wear out.

\section{SIMULATION RESULTS}

The simulation experiment compares the results obtained in the tuning of the two PID controllers in an independent manner (that is, SISO tuning) and those obtained in their simultaneous tuning (MIMO tuning). 
In this study, the process simulator described by (1) has two controlled and two manipulated variables:

$$
A=\left[\begin{array}{cc}
-1 & 0.1 \\
-0.2 & -0.3
\end{array}\right] ; \quad B=\left[\begin{array}{cc}
1 & -0.1 \\
0.01 & 0.03
\end{array}\right] .
$$

The tuning is performed within a time horizon of 150 elements of $600 \mathrm{~ms}$ each $(\Delta t=0.6 \mathrm{~s})$. The setpoint for variable $y_{1}$ is 1.0 before element 50 and 0.5 afterwards. The setpoint for variable $y_{2}$ is 0.75 before element 100 and 1.2 afterwards. In all of the experimental runs, the weights in (3) are $1.0\left(w_{1}=w_{2}=\left[\begin{array}{ll}1.0 & 1.0\end{array}\right]\right)$.

\section{SISO tuning}

In this setup, each of the PID controllers is tuned independently. The manipulated variable of the other controller is maintained at a fixed value. The resulting closed loop responses for the situations used in the SISO procedure are presented in Figure 3.
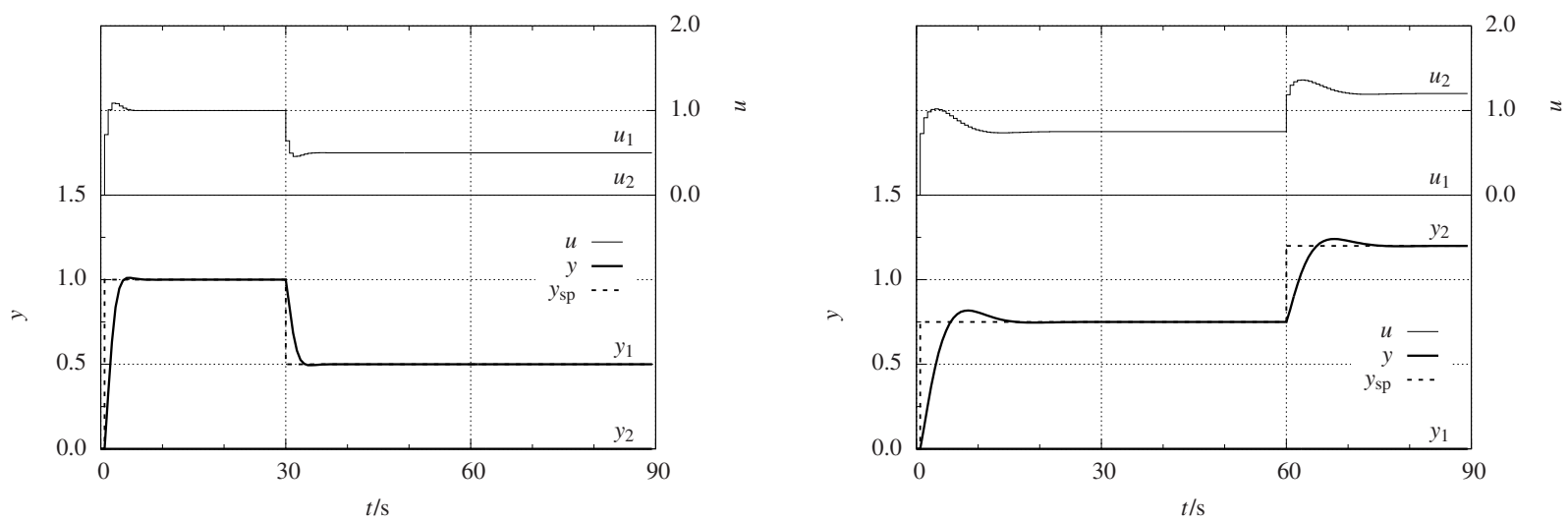

FIGURE 3. Closed loop responses for the SISO tuning procedure.

The resulting tuning parameters are summarized in Table 1(a).

TABLE 1. Tuning parameters.

(a) SISO case.

\begin{tabular}{llll}
\hline Parameter & Loop 1 & Loop 2 & Units \\
\hline$K_{\mathrm{c}}$ & 0.3890625 & 0.7226806 & {$[u] /[y]$} \\
$\tau_{\mathrm{I}}$ & 0.7537841 & 1.7482910 & $\mathrm{~s}$ \\
$\tau_{\mathrm{D}}$ & 0.0252685 & 0.0 & $\mathrm{~s}$ \\
\hline
\end{tabular}

(b) MIMO case.

\begin{tabular}{llll}
\hline Parameter & Loop1 & Loop2 & Units \\
\hline$K_{\mathrm{c}}$ & 0.3915039 & 0.7203613 & {$[u] /[y]$} \\
$\tau_{\mathrm{I}}$ & 0.8005371 & 1.1180419 & $\mathrm{~s}$ \\
$\tau_{\mathrm{D}}$ & 0.0 & 0.0 & $\mathrm{~s}$ \\
\hline
\end{tabular}

\section{MIMO tuning}

The multivariable nature of the process is taken into account in the case of multivariable tuning where both controllers are in automatic mode. The resulting closed-loop response for the situation used in the tuning procedure is shown in Figure 4 and the tuning parameters are given in Table 1(b).

Given the linear nature of the process, the parameters obtained via SISO and MIMO tuning are similar, particularly, the controller gains. However, the integral time constants show a tangible difference that can be attributed to the interactivity between the controlled and manipulated variables of the loops.

The value of $\tau_{\mathrm{D}}$ is zero in order to minimize the error. In this particular case, the system is linear and has no deadtime. Therefore, the derivative action (nonzero $\tau_{\mathrm{D}}$ ) would unnecessarily slow down the closed loop dynamics that would increase the overall setpoint tracking error. The value of zero corresponds to the industrial practice in such systems. 


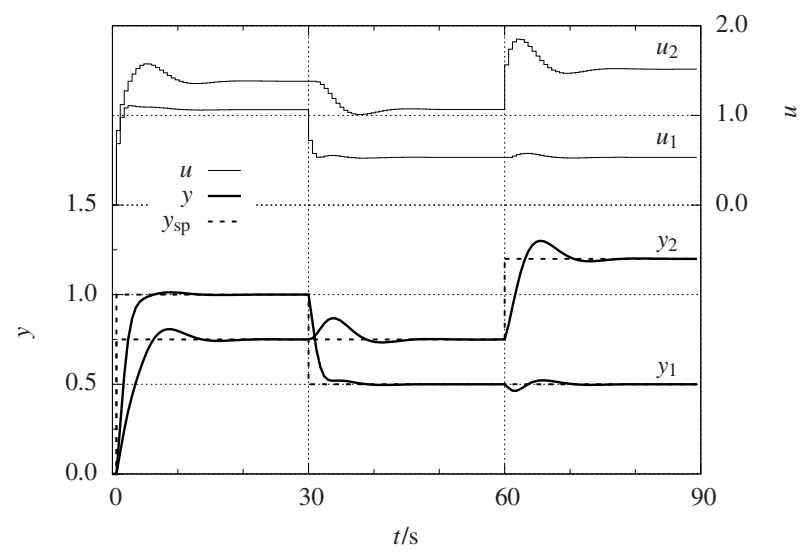

FIGURE 4. Closed loop response for the MIMO tuning procedure.

\section{CONCLUSIONS}

PID controllers are the bulk option in industrial plants, but they require a proper tuning. Optimization techniques can be advantageously used for this purpose compared to traditional methods. The tuning task was cast as a bounded optimization problem whose design was based on two different perspectives of the system: SISO and MIMO approaches. The minimization problems were solved by means of the MCSFilter algorithm and the found minimizers allow an adequate dynamic response of the system operating in closed loop.

\section{REFERENCES}

[1] L. Eriksson and H. Koivo, "Tuning of discrete-time pid controllers in sensor network based control systems," in Computational Intelligence in Robotics and Automation, 2005. CIRA 2005. Proceedings. 2005 IEEE International Symposium on (2005), pp. 359 - 364.

[2] McMillan G.K., "PID Control in the Third Millennium. Advances in Industrial Control," (Springer, 2012) Chap. Industrial Applications of PID Control.

[3] Antonio Visioli, Acta Polytechnica 52, 144-150 (2012).

[4] Terrence L. Blevins, "PID Advances in Industrial Control," in Proceedings of IFAC Conference on Advances in PID Control - PID'12 (2012) pID'12 Brescia (Italy), March 28-30, 2012.

[5] J. Ziegler and N. Nichols, Transactions of the ASME Journal 64, $759-768$ (1942).

[6] G. H. Cohen and G. A. Coon, Transactions of the ASME 75, 827 - 834 (1953).

[7] W. L. Luyben, Ind. Eng. Chem. Proc. Des. Devel. 25, p. 654 ff (1986).

[8] C. Hang, K. Åström, and W. Ho, Control Theory and Applications, IEE Proceedings D 138, $111-118$ (1991).

[9] T. S. Schei, Automatica 30, 1983 - 1989 (1994).

[10] Z. Palmor, Y. Halevi, and N. Krasney, Automatica 31, 1001 - 1010 (1995).

[11] R. Dittmar, S. Gill, H. Singh, and M. Darby, Control Engineering Practice 20, 355 - 370 (2012), special Section: IFAC Symposium on Advanced Control of Chemical Processes - ADCHEM 2009.

[12] F. P. Fernandes, M. F. P. Costa, and E. M. G. P. Fernandes, "Multilocal programming: A derivative-free filter multistart algorithm," in Computational Science and Its Applications - ICCSA 2013: 13th International Conference, Ho Chi Minh City, Vietnam, June 24-27, 2013, Proceedings, Part I, edited by B. Murgante, S. Misra, M. Carlini, C. M. Torre, H.-Q. Nguyen, D. Taniar, B. O. Apduhan, and O. Gervasi (Springer Berlin Heidelberg, Berlin, Heidelberg, 2013), pp. 333-346.

[13] D. E. Seborg, T. F. Edgar, and D. Mellichamp, Process Dynamics and Control, 2nd ed. (John Wiley \& Sons, Inc., 2003). 